\title{
X-RAY CONSTRAINTS ON AGN CLUSTERING
}

\author{
G. De Zotti ${ }^{1}, M$. Persic $^{2}$, A. Franceschini ${ }^{1}$, L. Danese $^{1}$, \\ G.G.C. Palumbo ${ }^{3}$, E.A. Boldt ${ }^{2}$, and F.E. Marshall ${ }^{2}$
}

1 Osservatorio Astronomico and Dipartimento di Astronomia, Padova, Italy

2 Laboratory for High Energy Astrophysics, NASA/GSFC

3 Dipartimento di Astronomia, Bologna, Italy

Studies of the HEAO-1 A2 all-sky survey data have established that the level of anisotropy of the extragalactic X-ray background (XRB) is relatively low:

- The cell-to-cell XRB intensity variations can be entirely accounted for by Poisson fluctuations in the space distribution of known classes of sources; the $90 \%$ confidence upper limit to any additional contribution on a scale of 26 square degrees is $2.3 \%$ (Shafer and Fabian 1983).

- No significant correlations of XRB intensity fluctuations appear to be present; the formal $90 \%$ confidence upper limit on the amplitude of autocorrelations, relative to the mean background intensity, for an angular scale of $3^{\circ}$ is $\Gamma\left(3^{\circ}\right) \leq 1.9 \times 10^{-2}$ (Persic et al. 1988).

The above results entail significant constraints on clustering properties of hard $\mathrm{X}$-ray sources. The figure shows the predicted contributions of Active Galactic Nuclei (AGNs) to autocorrelations on a scale $\theta=3^{\circ}$ (upper panels) and to fluctuations on a scale of 26 square degrees (lower panels) for the case of a power-law two-point spatial correlation function

$$
\begin{gathered}
\xi(r, z)=\xi_{0}(r)(1+z)^{-(3+\epsilon)}, \\
\xi_{0}(r)= \begin{cases}\left(r / r_{0}\right)^{-1.8}, & \text { if } r<r_{\max } \\
0, & \text { if } r \geq r_{\max }\end{cases}
\end{gathered}
$$

as a function of the clustering length $r_{0}$.

The cutoff radius $r_{\text {max }}$ takes into account that $\xi_{0}(r)$ will steepen and eventually become negative at large distances.

The last factor in eq. (1) models the possible cosmological evolution of clustering. If the number of objects at fixed proper separation $r, n(z) \xi(r, z)$, is constant (statistically stable clustering), $\epsilon=0$; if $\xi(r, z)$ is constant in physical coordinates, $\epsilon=-3$.

\section{References}

Persic, M., De Zotti, G., Boldt, E.A., Marshall, F.E., Danese, L., Franceschini, A., and Palumbo, G.G.C. 1988, Ap. J. Lett., submitted.

Shafer, R.A., and Fabian, A.C. 1983, in IAU Symp. No. 104, p. 333. 492 


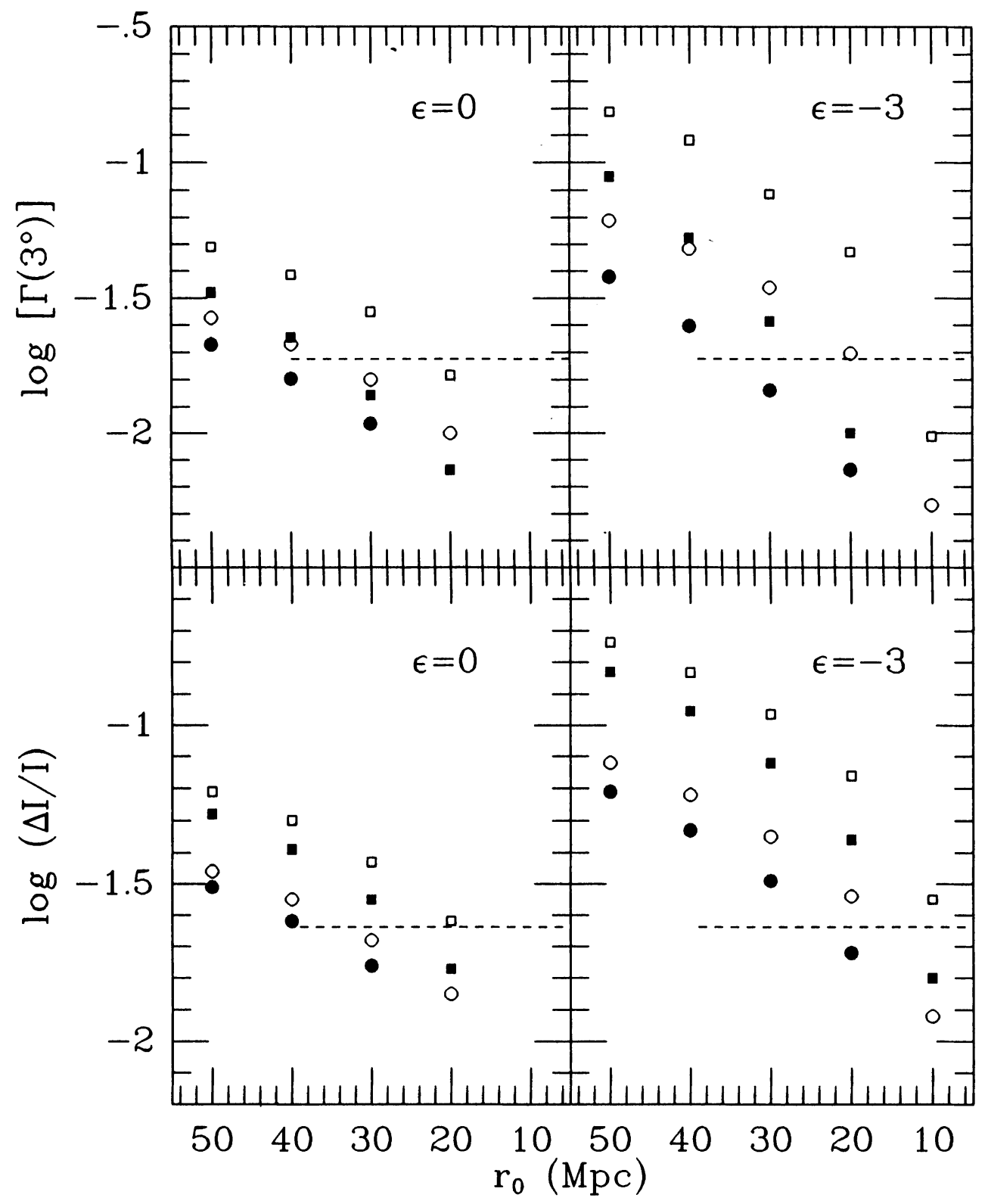

Predicted 2-10 keV contributions of AGNs to autocorrelations on a scale of $3^{\circ}$ (upper panels) and to non-Poisson fluctuations (lower panels) on a scale of 26 square degrees, for the cases $\epsilon=0$ and $\epsilon=-3$. The squares refer to pure luminosity evolution, the circles to the case that only sources with $\log L_{x}>43.5$ evolve in luminosity. The open symbols correspond to $r_{\max }=6 r_{0}$, the filled ones to $r_{\max }=2 r_{0}$. The dashed lines show the $90 \%$ confidence upper limits. We have assumed $H_{0}=50$ and $\Omega=1$. 